\title{
Model for the static friction coefficient in a full stick elastic-plastic coated spherical contact
}

\author{
Zhou CHEN", Izhak ETSION \\ Department of Mechanical Engineering, Technion, Haifa 32000, Israel \\ Received: 15 July 2018 / Revised: 26 August 2018 / Accepted: 15 October 2018 \\ (C) The author(s) 2018. This article is published with open access at Springerlink.com
}

\begin{abstract}
Finite element analysis is used to investigate an elastic-plastic coated spherical contact in full stick contact condition under combined normal and tangential loading. Sliding inception is associated with a loss of tangential stiffness. The effect of coating thickness on the static friction coefficient is intensively investigated for the case of hard coatings. For this case, with the increase in coating thickness, the static friction coefficient first increases to its maximum value at a certain coating thickness, thereafter decreases, and eventually levels off. The effect of the normal load and material properties on this behavior is discussed. Finally, a model for the static friction coefficient as a function of the coating thickness is provided for a wide range of material properties and normal loading.
\end{abstract}

Keywords: contact mechanics; elastic-plastic contact; spherical contact; static friction; hard coatings

\section{Introduction}

Friction is ubiquitous in our lives. On one hand, it can be desirable in some cases such as a braking system [1] and power transmission system [2,3]. On the other hand, it may not be favored as high friction can lead to severe material wear [4] and undesirable energy consumption [5]. Thus, proper control of friction is an essential goal for the surface engineering community.

Coating technology has been widely used in the industry and has been proven to be one of the most effective surface treatments to control the surface friction property [6-8]. However, the selection of some important parameters, such as coating thickness and coating material, in coating applications still mainly relies on empiricism [9]. A generic scientific theory is thus required so that the surface properties can be tailored precisely with less trial and error.

In numerous theoretical studies on surface contact models, a nominally flat surface is envisaged as a cluster of asperities, whose heights have some statistical distribution (e.g., the classic GW model [10]). The contact between surfaces is determined by statistically incorporating the individual asperity contact behavior. If we assume that the coating inherits the topography of the substrate and the asperities on the substrate surface have spherical shape, the contact between coated surfaces is localized to many individual coated spherical tips. With this assumption, understanding the behavior of a single coated sphere under combined normal and tangential loading is the first step for the establishment of a theory on the friction property of a coated surface.

The behavior of a coated sphere under pure normal loading has been intensively investigated in various aspects of both elastic [11-18] and elastic-plastic [19-25] regimes. Keer et al. [11] obtained the stress distribution at the contact interface of two identical coated spheres. Under the same contact radius, a stiffer coating produces higher interfacial normal stress. Garjonis et al. [12] determined an equivalent modulus of elasticity for identical contacting coated spheres that is load dependent. However, in practice, it may be inconvenient to use such an effective modulus

* Corresponding author: Zhou CHEN, E-mail: chenzhouhit@gmail.com 


$\begin{array}{llll}\text { Nomenclature } & \\ E & \text { Young's modulus } & \omega & \text { Interference } \\ L_{\mathrm{c}} & \text { Critical load of a homogeneous sphere under } & u & \text { Tangential displacement } \\ & \text { the stick condition } & \text { Subscripts } \\ P & \text { Dimensional contact load } & \text { co } & \text { Coating } \\ P^{*} & \text { Dimensionless contact load, } P /\left(E_{\mathrm{su}} R^{2} \times 10^{-7}\right) & \mathrm{su} & \text { Substrate } \\ R & \text { Radius of the spherical substrate } & \mathrm{m} & \text { Corresponds to the maximum static friction } \\ Y & \text { Yield strength } & & \text { coefficient }\end{array}$

owing to its load dependency. This inconvenience was resolved by Goltsberg and Etsion [13], who derived a load-independent effective elasticity modulus based on their extensive numerical results. They also introduced a special normalization for contact parameters that enabled universal dimensionless relations for loaddisplacement [13] and displacement-contact area [14] in elastic regime.

Another important aspect of the elastic deformation in a coated sphere owing to normal loading is its elasticity terminus. Goltsberg et al. [15] showed various possible locations of yield inception, which can be in the coating, in the substrate, or at their interface depending on the coating thickness. Chen et al. [16] extended this analysis by presenting the location of yield inception in a yield map as a function of both coating thickness and material properties of the coating and substrate. In addition, Goltsberg and Etsion [17] pointed out a weakening effect associated with very thin hard coatings where the resistance of the coated sphere to plastic yield is even lower than that of the homogeneous sphere made of the weaker substrate material. This weakening effect was experimentally validated by Huang et al. [18].

Compared with the elastic deformation in a coated sphere, the elastic-plastic deformation is inevitable and may be beneficial in practice, which, for instance, can generate sufficient contact area to establish a reliable electrical contact [19-22]. Both numerical [19, 20] and experimental studies [21,22] were conducted to obtain the load-displacement and load-contact area relationships. However, these results were obtained only for a few specific material properties and geometric dimensions. To overcome this limitation, a wide range of material properties and coating thicknesses were considered in [23-25]. Chen et al. [23] showed the plasticity evolution in a coated sphere loaded by a rigid flat under the slip contact condition, where the coating thickness was selected to avoid the weakening effect [17]. After the first yield inception, a second yield inception occurred on the substrate side of the interface. Subsequently, they normalized the contact parameters by critical values at the second yield inception and obtained universal dimensionless relations for load-displacement and contact area-displacement in elastic-plastic regime [24]. Ronen et al. [25] compared the plasticity evolution and contact parameters under slip and stick conditions and observed that the effect of contact condition was negligible except for a phenomenon occurring close to the contact area.

Despite the considerable number of studies on the behavior of a coated sphere under pure normal loading, studies concerning combined normal and tangential loading are relatively scant and are limited to the elastic regime. Keer et al. [11] presented the shear stress distribution at the contact interface of two identical coated spheres elastically deformed under the combined loading. They assumed that an increase in tangential loading would trigger the occurrence of local slip at the originally bonded interface once the Coulomb friction law is satisfied. Such a local slip is initiated from the contact edge, and thereafter, it progresses radially inward until a global interfacial sliding. Based on this assumption, they further observed that the friction force is independent of coating thickness.

The studies concerning combined normal and tangential loading in elastic-plastic regime were mostly conducted for a homogeneous sphere. To avoid the empiricism-based Coulomb friction law, many 
researchers sought to associate the friction property with various material properties such as yield strength and toughness [26-35]. Partial slip was allowed at the interface in Refs. [26-31] whereas the full stick condition was maintained during tangential loading in Refs. [32-35]. Although allowing partial slip is realistic under low to moderate normal loading, it is still difficult to achieve a satisfying law governing partial slip owing to the complex interfacial bonding contributed by multiple intertwined physical and chemical sources [31]. The much simplified extreme case of full stick condition is an appropriate description of interfacial bonding [36]. Under high normal loading, the full stick condition was experimentally demonstrated to be maintained throughout the loading process [37]. Under low to moderate normal loading, the full stick condition also correlates well with the slip occurrence at the contact interface as the plastic volume resulting from the combined loading is confined to close proximity below this interface, indicating that the slip occurs at the interface or very close to it. Thus, the simpler full stick condition [32-35] will be adopted in the present study.

Brizmer et al. [32] observed that an increase in the tangential load leads to a decrease in the tangential stiffness. They assumed that this stiffness finally disappears at sliding inception. By defining the tangential force at sliding inception as the static friction force, they obtained the static friction coefficient as a decreasing function of normal loading. Additionally, Zolotarevskiy et al. [33] showed the evolution of tangential force from the initiation of tangential loading to sliding inception. In both Refs. [32] and [33], the material is bilinear elastic-plastic with $2 \%$ tangent modulus of isotropic linear hardening. Bhagwat et al. [34] reported that a larger tangent modulus results in a higher friction coefficient. Zhao et al. [35] performed a similar study but on materials with power-law hardening as it is a more realistic description of behavior for many materials.

From the above literature review, it can be observed that the studies on coated spherical contact have been conducted so far with pure normal loading. Studies on combined normal and tangential loading are limited to elastic regime only and rely on Coulomb friction law (e.g., [11]). Therefore, the aim of the present study is to investigate the elastic-plastic coated spherical contact under combined normal and tangential loading with the full stick contact condition and a power-law hardening.

\section{Static friction coefficient of a homogeneous spherical contact [32]}

Figure 1 schematically shows a deformable coated sphere in contact with a rigid flat under combined normal and tangential loading. Notably, indentation is often used for characterizing mechanical properties of coating but may be detrimental in sliding systems. This is because the indenting asperities of a rough surface may result in severe abrasive friction and wear owing to plowing. Hence, for a good tribological design, indentation of asperities should be avoided and flattening of asperities associated with mild adhesive friction and wear should be attempted. Loading of the coated sphere is achieved by first applying a normal load $P$, and subsequently a tangential displacement $u_{x}$. The special case where the coating and substrate materials are identical is a homogeneous spherical contact. This contact problem under the full stick condition has been intensively investigated in Ref. [32]. Upon the completion of normal loading, a contact area is established at the contact interface. Subsequently, tangential displacement $\left(u_{x}\right)_{i}$ increased in a stepwise manner is applied, where $i$ is the number of consecutive tangential displacement steps. The corresponding tangential force $(Q)_{i}$ can be obtained as the $x$ component of the reaction force at the sphere bottom. Accordingly, the tangential stiffness of the junction $\left(K_{\mathrm{T}}\right)_{i}$ can be approximated as

$$
\left(K_{\mathrm{T}}\right)_{i}=\left(\frac{\partial Q}{\partial u_{\mathrm{x}}}\right)_{i} \approx \frac{Q_{i}-Q_{i-1}}{\left(u_{\mathrm{x}}\right)_{i}-\left(u_{\mathrm{x}}\right)_{i-1}}
$$

It decreases with the tangential loading owing to the accumulated plasticity in the junction. It was assumed in Ref. [32] that, once $\left(K_{\mathrm{T}}\right)_{i}$ becomes equal to or less than $10 \%$ of the initial tangential stiffness $\left(K_{\mathrm{T}}\right)_{1}$, i.e., $\left(K_{\mathrm{T}}\right)_{i} \leq 0.1\left(K_{\mathrm{T}}\right)_{1}$, the junction cannot support significant additional tangential force and sliding inception occurs. Hence, sliding inception is treated as the junction plastic failure. The corresponding tangential force is the maximum tangential force $Q_{\max }$ that can be supported by the junction and is defined as the 
static friction force. Accordingly, the static friction coefficient $\mu$ is $Q_{\max } / P$.

An empirical expression for $\mu$ was obtained in Ref. [32] as a function of the dimensionless normal load $P / L_{\mathrm{c}}$

$$
\mu=0.27 \operatorname{coth}\left(0.27\left(P / L_{c}\right)^{0.35}\right)
$$

where $L_{c}$ is the critical normal load at yield inception of a homogeneous sphere under the stick condition in the form

$$
L_{\mathrm{c}}=\bar{L}_{\mathrm{c}} \frac{\pi^{3} Y}{6} C_{v}{ }^{3}\left[R\left(1-v^{2}\right) \frac{Y}{E}\right]^{2},
$$

where $v, E$, and $Y$ are the Poisson's ratio, Young's modulus, and yield strength of the material, respectively, and $\bar{L}_{\mathrm{c}}=8.88 v-10.13\left(v^{2}+0.089\right), C_{v}=1.234+1.256 v$. From Eq. (2), the static friction coefficient $\mu$ decreases with the increase in normal load $P$. This is physically reasonable as a higher normal load $P$ results in a more plastic and compliant junction that can support a lower dimensionless tangential load $Q_{\max } / P$ [32].

\section{Finite element model}

A coated spherical contact under combined normal and tangential loading is schematically presented in Fig. 1, where the coated sphere is composed of a substrate of radius $R$ and a coating of thickness $t$. To solve this complex contact problem, the finite element method, implemented using the commercial software ANSYS 18.1, was used. Owing to the symmetry, the contact problem can be simulated by only a quarter of the coated sphere in contact with a rigid flat as presented in Fig. 2. The thick solid curve inside the coated sphere indicates the coating/substrate interface. The 3D mesh was generated by rotating the 2D mesh (i.e., the meshed quarter of a circle on the $x-z$ plane) by $180^{\circ}$ about the $z$-axis with 10 volumes [38]. The $2 \mathrm{D}$ mesh was divided into four different but fixed mesh density zones, where Zones I, II, and III were within $t+0.01 R, t+0.025 R$, and $t+0.1 R$, respectively, from the sphere tip and zone IV is outside the distance $t+0.1 R$. The four zones had a gradually coarser mesh with the increase in their distance from the sphere tip.

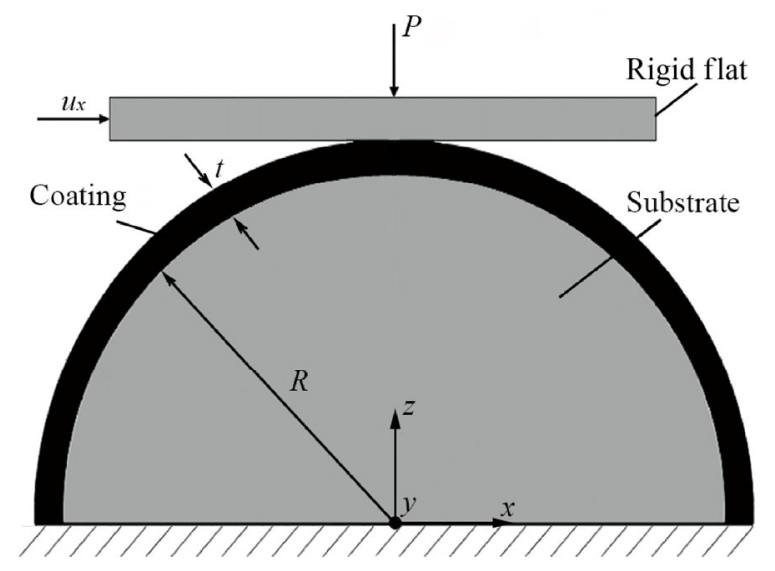

Fig. 1 Coated sphere under combined normal and tangential loading.

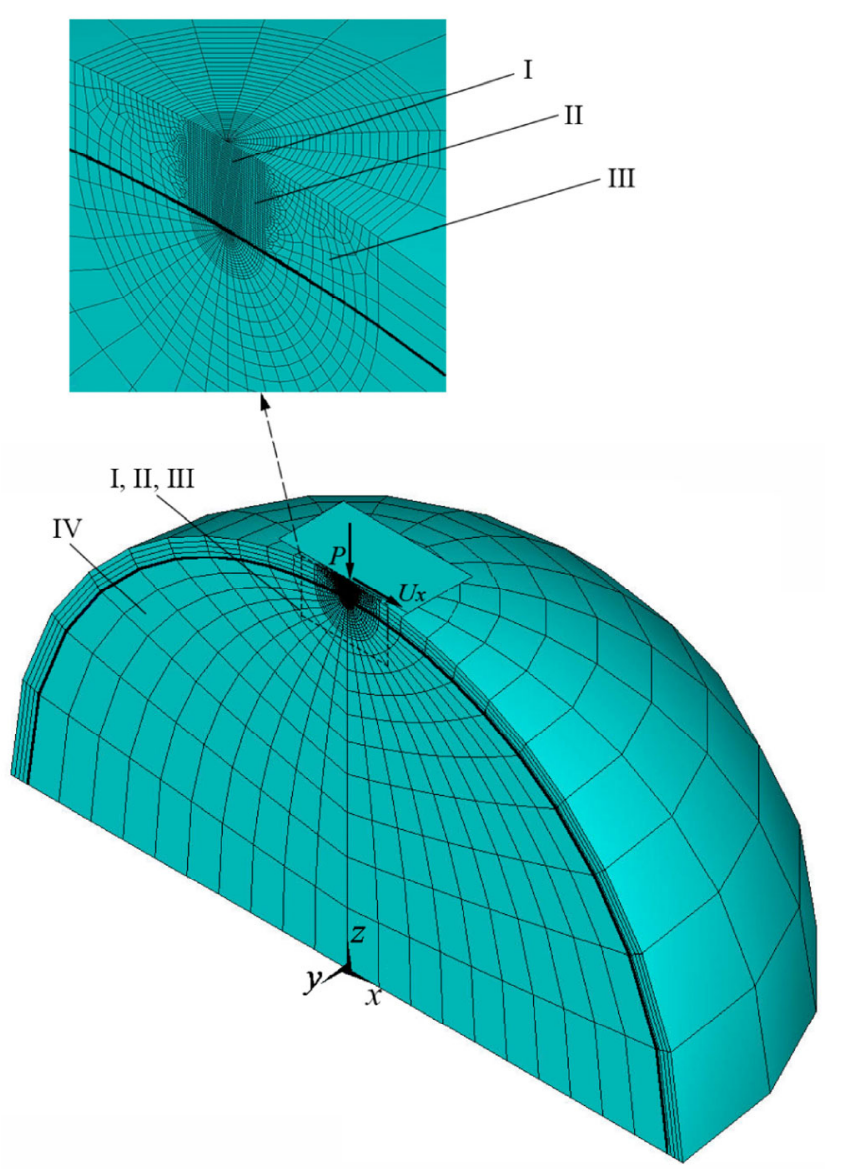

Fig. 2 Finite element model of the coated sphere under combined normal and tangential loading.

In general, the element size in Zones I to IV was $0.001 R, 0.0025 R, 0.01 R$, and $0.1 R$, respectively, whereas for extremely thin coatings, the element size in Zones I and II was adjusted to ensure at least 10 elements in the coating along the coating thickness. Consequently, 
the 3D mesh of the coated sphere contained 6,842 to 14,560 20-node brick-shaped elements (SOLID 186) depending on the coating thickness. 3-D 8-node contact elements (CONTA 174) and 3-D target elements (TARGE 170) were used to model the outer surface of the coated sphere and the rigid flat, respectively. The rectangular-shaped rigid flat had the dimensions of $0.4(R+t) \times 0.2(R+t)$ in the $x$ and $y$ directions, respectively, which were sufficient to cover the maximum possible contact area encountered in the present study.

The following main assumptions were adopted in the present study:

1) The stick contact condition exists between the outer surface of the coated sphere and the rigid flat.

2) The coating is perfectly bonded to the substrate.

3) The coating and substrate materials are homogeneous and isotropic.

4) The coating and substrate are free of residual stresses.

5) The Poisson's ratios for the coating and substrate materials are equal, i.e., $v_{\mathrm{co}}=v_{\mathrm{su}}=v=0.32$.

The coating and substrate materials are elastic-plastic and the transition from elastic to plastic deformation was determined using the von Mises yielding criterion. The stress-strain relations in the elastic and plastic zones were governed by the Hooke's law and the Prandtl-Reuss law with isotropic power-law hardening, respectively. The power-law hardening is adopted owing to its capability for better modeling the material behavior [35]. In uni-axial tension, the relation between the strain $\varepsilon$ and the stress $\sigma$ is

$$
\varepsilon=\left\{\begin{array}{l}
\sigma / E, \sigma<Y ; \\
(Y / E)(\sigma / Y)^{1 / n}, \sigma \geq Y
\end{array}\right.
$$

where $n$ is the strain hardening exponent. A larger $n$ indicates a stronger hardening effect. $n=0$ and $n=1$ represent the elastic-perfectly plastic case and purely elastic case, respectively. In the present study, a small value i.e., $n=0.01$ was selected for both coating and substrate materials.

The nodes on the $x-z$ plane were constrained in the $y$ direction owing to the symmetry. The nodes on the $x-y$ plane were constrained in all directions. Notably, the full constraint of bottom nodes will affect the results negligibly as the bottom is very far from the contact zone [23]. The point at the location $(0,0, R+t)$ on the rigid flat was selected as the pilot node [39], whose motion can govern that of the entire rigid flat. A constant normal load $P$ was first applied to the pilot node and the corresponding normal interference $\omega_{0}$ was obtained as the displacement of the summit point of the coated sphere. Subsequently, the tangential displacement $\left(u_{\mathrm{x}}\right)_{i}$ applied to the pilot node was increased in a stepwise manner. The corresponding tangential force $Q_{i}$ was obtained as the sum of the $x$ component of the reaction forces of nodes at the sphere bottom. Accordingly, the tangential stiffness $\left(K_{\mathrm{T}}\right)_{i}$ can be calculated using Eq. (1).

As in Ref. [32] (see Section 2), the sliding inception criterion for the coated spherical contact is also selected here as $\left(K_{\mathrm{T}}\right)_{i} \leq 0.1\left(K_{\mathrm{T}}\right)_{1}$. This criterion was justified by attempting lower values (e.g., $\left.\left(K_{\mathrm{T}}\right)_{i} \leq 0.05\left(K_{\mathrm{T}}\right)_{1}\right)$ that increased $\mu$ negligibly but at a much higher cost of computing time. From the sliding inception criterion, it is crucial to capture the initial tangential stiffness $\left(K_{\mathrm{T}}\right)_{1}$ accurately. As $K_{\mathrm{T}}$ decreases with $u_{\mathrm{x}}$ it is apparent that $\left(K_{\mathrm{T}}\right)_{1}$ obtained at a smaller $\left(u_{\mathrm{x}}\right)_{1}$ can better approximate the real initial tangential stiffness. Consequently, $\left(u_{\mathrm{x}}\right)_{1}$ was set as $0.001 \omega_{0}$, which is a very small displacement step compared with the following steps $\left(u_{\mathrm{x}}\right)_{i}-\left(u_{\mathrm{x}}\right)_{i-1}=0.05 \omega_{0}$ for $i \geq 2$. The use of a $\left(u_{\mathrm{x}}\right)_{1}$ smaller than $0.001 \omega_{0}$ yielded a negligible difference in $\left(K_{\mathrm{T}}\right)_{1}$.

To verify the accuracy of the present $3 \mathrm{D}$ finite element model, two groups of comparisons were made with the results obtained in the literature for simpler cases.

1) The use of identical materials for coating and substrate in the current model of the coated sphere facilitates the comparison with the results in a homogeneous spherical contact. Under pure normal loading in the slip contact condition, the present loadinterference and interference-contact area relations differ from those in the Hertz solution by less than $1 \%$ and $5 \%$, respectively. Under combined normal and tangential loading in the stick condition, the present tangential displacement-tangential load relations under a wide range of normal loads differ from those in Ref. [33] by less than $7 \%$.

2) The use of different materials for coating and substrate facilitates the comparison with the results in a coated spherical contact under pure normal loading. 
The load-interference and interference-contact area relations under the slip contact condition differ from those in Ref. [24] by less than $0.5 \%$ and $2 \%$, respectively, and those under the stick contact condition [25] by less than $4 \%$ and $5 \%$, respectively.

Finally, a convergence test was performed for the current model of the coated sphere under combined normal and tangential loading in the full stick condition by increasing the mesh density until a further increase affected the final results negligibly.

\section{Results and discussion}

With fixed $E_{\mathrm{su}}$ and $R$, the material and geometric properties of a coated sphere can be determined by four dimensionless parameters $E_{\mathrm{co}} / E_{\mathrm{su}}, E_{\mathrm{co}} / Y_{\mathrm{co},}, E_{\mathrm{su}} / Y_{\mathrm{su}}$ and $t / R$, where subscripts 'co' and 'su' indicate the coating and substrate material, respectively. In previous studies on an elastic-plastic coated spherical contact [23-25], relations between these four dimensionless parameters and various dimensionless tribological parameters under fixed $E_{\mathrm{su}}$ and $R$ were investigated. It was observed that these relations remain the same even for different $E_{\mathrm{su}}$ and $R$ as long as the above four dimensionless parameters characterizing the coated sphere remain fixed. Hence, these relations depend only on the dimensionless parameters.

Similarly, the present study was conducted with fixed $E_{\mathrm{su}}=200 \mathrm{GPa}$ and $R=10 \mathrm{~mm}$ and the results were also verified to be independent of $E_{\mathrm{su}}$ and $R$ as long as $E_{\mathrm{co}} / E_{\mathrm{su}}, E_{\mathrm{co}} / Y_{\mathrm{co},}, E_{\mathrm{su}} / Y_{\mathrm{su}}$ and $t / R$ and the dimensionless normal load (see Section 4.1) were fixed. Thus, the dimensionless results discussed in Sections 4.1 to 4.3 also depend only on the dimensionless parameters.

\subsection{Effect of geometric parameter $t / R$}

Figure 3 presents the static friction coefficient $\mu$ as a function of $t / R$ when $E_{\mathrm{co}} / E_{\mathrm{su}}=4, E_{\mathrm{co}} / Y_{\mathrm{co}}=E_{\mathrm{su}} / Y_{\mathrm{su}}=1,000$ under the dimensionless normal load $P^{*}=50 . P^{*}$ is defined as $P /\left(E_{\mathrm{su}} R^{2} \times 10^{-7}\right)$. As $E_{\mathrm{su}}$ and $R$ are fixed, the dimensional normal load $P$ is proportional to $P^{*}$. Thus, the results in Fig. 3 are obtained under the same dimensional normal load $P$. Therefore, only the effect of the geometric parameter $t / R$ on the static friction coefficient is revealed in Fig. 3.

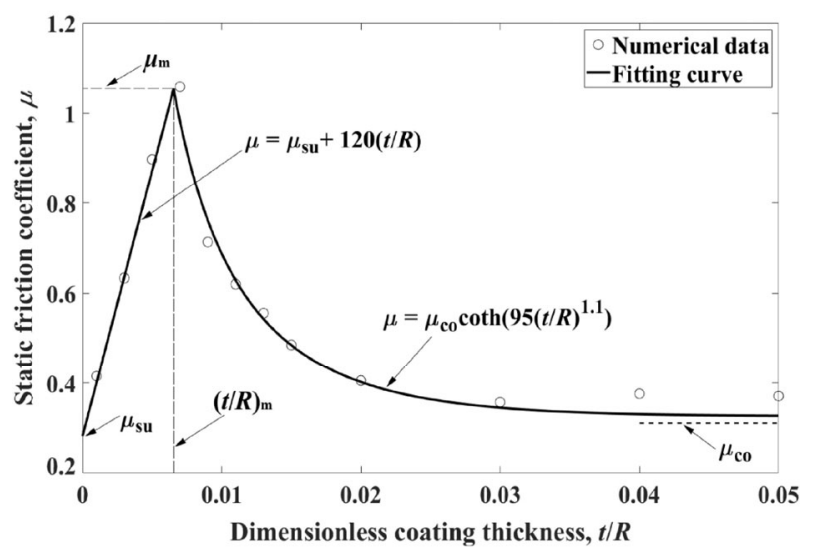

Fig. 3 Static friction coefficient $\mu$ as a function of $t / R$ for $E_{\mathrm{co}} / E_{\mathrm{su}}=4, E_{\mathrm{co}} / Y_{\mathrm{co}}=E_{\mathrm{su}} / Y_{\mathrm{su}}=1,000$ and $P^{*}=50$.

It can be observed that the static friction coefficient $\mu$ first increases linearly with $t / R$ from $\mu_{\mathrm{su}}$ at $t / R=0$ till reaching a maximum $\mu_{\mathrm{m}}$ at $(t / R)_{\mathrm{m}}$. A further increase in $t / R$ above $(t / R)_{\mathrm{m}}$ leads to a decrease in $\mu$, which eventually approaches $\mu_{\mathrm{co}}$. The static friction coefficients $\mu_{\mathrm{su}}$ and $\mu_{\mathrm{co}}$ of a homogeneous spherical contact can be calculated by first obtaining the corresponding dimensional load $P=P^{*}\left(E_{\mathrm{su}} R^{2} \times 10^{-7}\right)$ and subsequently substituting $P$ into Eq. (2). Finally, the expressions for $\mu_{\mathrm{su}}$ and $\mu_{\mathrm{co}}$ are as follows:

$$
\begin{gathered}
\mu_{\mathrm{su}}=0.27 \operatorname{coth}\left(0.27\left(P^{*} E_{\mathrm{su}} R^{2} \times 10^{-7} / L_{\mathrm{c} \_ \text {su }}\right)^{0.35}\right) \\
\mu_{\mathrm{co}}=0.27 \operatorname{coth}\left(0.27\left(P^{*} E_{\mathrm{su}} R^{2} \times 10^{-7} / L_{\mathrm{c} \_ \text {co }}\right)^{0.35}\right)
\end{gathered}
$$

where $L_{\mathrm{c}_{-} \text {su }}$ and $L_{\mathrm{c}_{-} \text {co }}$ are the critical loads of a homogeneous sphere made of the substrate and coating material, respectively, under the stick condition (see Eq. (3)).

To explain the behavior of the static friction coefficient $\mu$ in Fig. 3, the relative contributions of the substrate and coating to the total tangential displacement $u_{\mathrm{x}}$ of the coated sphere should be investigated. A similar approach was used in Ref. [13] to explain a transition interference from the substrate to coating under normal loading. It was observed in Ref. [13] that the substrate is the dominant contributor to the interference at small $t / R$ whereas the coating is the dominant contributor at large $t / R$. Consequently, the yield inception is more likely to occur in the substrate for small $t / R$ and in the coating for large $t / R$ $[15,16]$. 
The tangential displacement of the highest point in the substrate $u_{\mathrm{su}}$ describes the contribution of the substrate to $u_{\mathrm{x}}$. The contribution of the coating $u_{\mathrm{co}}$ is thus $u_{\mathrm{x}}-u_{\mathrm{su}}$. Figure 4 presents $u_{\mathrm{su}} / u_{\mathrm{x}}$ at sliding inception as a function of $t / R$ for the case in Fig. 3. As shown in Fig. 4, the variation of $u_{\mathrm{su}} / u_{\mathrm{x}}$ with $t / R$ has three distinct stages (see the two vertical solid lines). In the first stage from 0 to $(t / R)_{\mathrm{m}}, u_{\mathrm{su}} / u_{\mathrm{x}}$ is almost 1 and hence, $u_{\mathrm{su}}$ is the sole contributor to $u_{\mathrm{x}}$. In the second stage where $t / R$ is from $(t / R)_{\mathrm{m}}$ to approximately $t / R=0.03$, $u_{\mathrm{su}} / u_{\mathrm{x}}$ decreases sharply with $t / R$. It drops to 0.5 at approximately $t / R=0.12$, where the coating and substrate contribute equally to $u_{\mathrm{x}}$. For $t / R$ above 0.12 , the contribution of the coating to $u_{\mathrm{x}}$ is greater. In the last stage, $u_{\mathrm{su}} / u_{\mathrm{x}}$ finally levels off at approximately 0.1 and the coating becomes the sole contributor to $u_{\mathrm{x}}$. The variation of $u_{\mathrm{su}} / u_{\mathrm{x}}$ with $t / R$ indicates that the behavior of a coated sphere with very small or very large $t / R$ approaches that of a homogeneous sphere made of the substrate or coating material, respectively, as expected. This correlates well with the behavior of the static friction coefficient at very small or very large $t / R$ in Fig. 3.

To explain the transitional behavior of the static friction coefficient in the intermediate range of $t / R$, the substrate plasticity level and contact area as a function of $t / R$ should be investigated. In Ref. [24], it was observed that a thicker hard coating, i.e., a higher $t / R$, better protects the substrate from plastic deformation and also results in a smaller contact area at the outer surface of the coating. As indicated in Section 2, based on Ref. [32], the sliding inception is a junction plastic failure. A more plastic junction can support a lower dimensionless tangential load $Q_{\max } / P$. Similarly, the von Mises equivalent stress level in a junction at sliding inception cannot exceed the yield strength of the material. A junction of smaller contact area with a higher normal stress level under the same normal load can support less additional shear stress, and hence, less friction force.

Figures 5 and 6 present the dimensionless plastic volume $V / V_{0}$ in the substrate and dimensionless contact area $A / A_{0}$ upon the completion of normal loading, respectively, as a function of $t / R$ for the case in Fig. 3. Here, $V_{0}$ and $A_{0}$ are the plastic volume in a homogeneous sphere made of the substrate material and the contact area in a homogeneous sphere made of

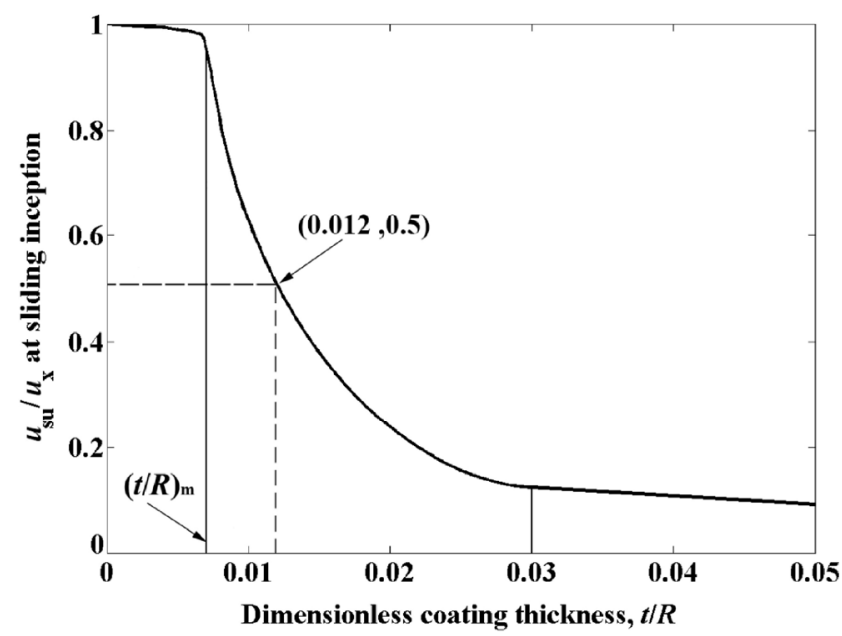

Fig. $4 u_{\mathrm{su}} / u_{\mathrm{x}}$ at sliding inception as a function of $t / R$ for the case in Fig. 3.

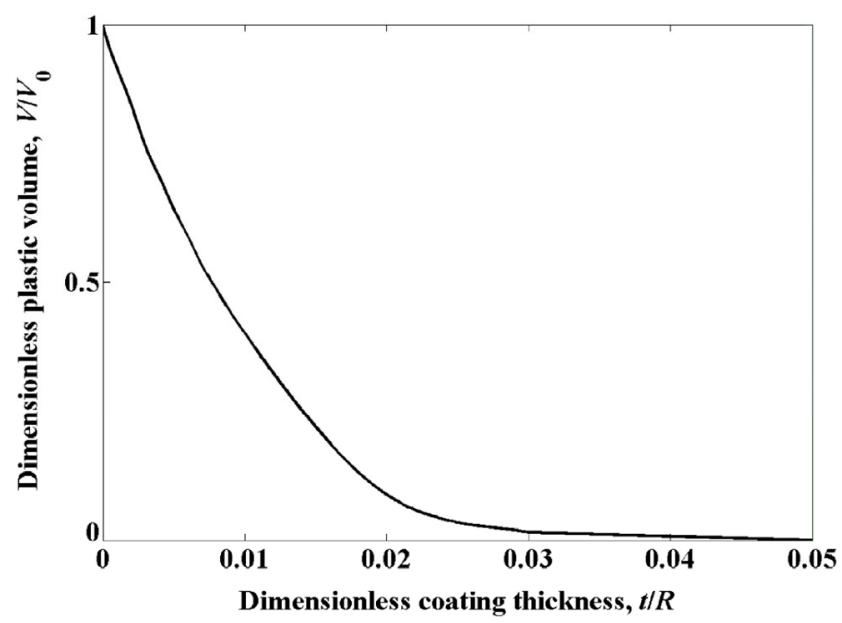

Fig. 5 Dimensionless plastic volume $V / V_{0}$ in the substrate upon the completion of normal loading as a function of $t / R$ for the case in Fig. 3.

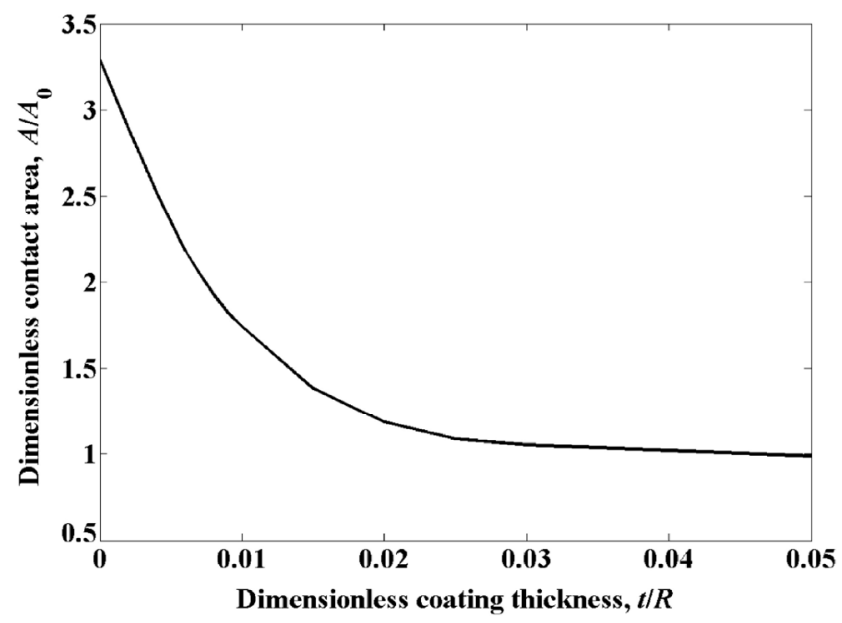

Fig. 6 Dimensionless contact area $A / A_{0}$ upon the completion of normal loading as a function of $t / R$ for the case in Fig. 3. 
the coating material under the same normal load, respectively. Both the substrate plasticity level $V$ and the contact area $A$ decrease with the increase in $t / R$ as expected.

The transitional behavior of the static friction coefficient in the intermediate range of $t / R$ can be attributed to the competition between two mechanisms. The first is that a decrease in the substrate plasticity level tends to increase $\mu$ and the second is that a decrease in the contact area tends to decrease $\mu$. For $t / R$ from 0 to $(t / R)_{\mathrm{m}}$, where the substrate is the dominant contributor to the tangential displacement $u_{\mathrm{x}}$ it is reasonable to assume that the first mechanism prevails. Hence, an increase in $t / R$ will increase the static friction coefficient. For $t / R$ values above $(t / R)_{\mathrm{m}}$, where the coating becomes the dominant contributor, it is reasonable to assume that the second mechanism prevails and hence, an increase in $t / R$ will decrease the static friction coefficient. In the small range just above $(t / R)_{\mathrm{m}}$, where the contribution of the substrate to $u_{\mathrm{x}}$ dramatically drops but $u_{\mathrm{su}} / u_{\mathrm{x}}$ still remains above 0.5 (see Fig. 3), it appears that the second mechanism involving a decrease in the contact area overcomes the first one involving a decrease in the substrate plasticity level. Hence, in this range, an increase in $t / R$ will decrease the static friction coefficient. An accurate quantitative validation of these assumptions is out of the scope of the present paper but can be addressed in future work.

\subsection{Effect of normal loading $P^{*}$ and material pro- perties $E_{\mathrm{co}} / E_{\mathrm{su},}, E_{\mathrm{co}} / Y_{\mathrm{co},} E_{\mathrm{su}} / Y_{\mathrm{su}}$}

To reveal the effect of each of these parameters on the static friction coefficient, a parametric study was performed based on the reference case of Fig. 3. This was performed by varying one parameter each time while maintaining the others the same as those in the reference case. The value of $t / R$ was selected from 0.001 to 0.05 as in Fig. 3. The dimensionless material parameters were selected as $2 \leq E_{\mathrm{co}} / E_{\mathrm{su}} \leq 8,500 \leq E_{\mathrm{su}} /$ $Y_{\mathrm{su}} \leq 2000$, and $500 \leq E_{\mathrm{co}} / Y_{\text {со }} \leq 2000$. As the present study was limited to hard coatings only, it was required that the ratio $Y_{\mathrm{co}} / Y_{\mathrm{su}}=\left(E_{\mathrm{su}} / Y_{\mathrm{su}}\right)\left(E_{\mathrm{co}} / E_{\mathrm{su}}\right) /\left(E_{\mathrm{co}} / Y_{\mathrm{co}}\right)$ be larger than 1 . This eliminated the combinations of low $E_{\mathrm{co}} / E_{\mathrm{su}}$ and $E_{\mathrm{su}} / Y_{\mathrm{su}}$ along with high $E_{\mathrm{co}} / Y_{\mathrm{co}}$ (see Ref. [16]). $P^{*}$ was selected from 20 to 100 so that the deformation of a coated sphere caused by $P^{*}$ is elastic-plastic for most combinations of $E_{\mathrm{co}} / E_{\mathrm{su}}, E_{\mathrm{co}} / Y_{\mathrm{co}}, E_{\mathrm{su}} / Y_{\mathrm{su}}$ and $t / R$ values in their ranges. Exceptional combinations are those with very large $t / R$, where even $P^{*}=100$ can only elastically deform the coated sphere. Nonetheless, the present study focuses on an elastic-plastic contact.

Figure 7 shows the effect of the normal loading. The static friction coefficient at small or large $t / R$ decreases with the increase in normal load (e.g., $\mu$ as a function of $P^{*}$ at $t / R$ denoted by the vertical dashed lines I and III). This is expected because the coated sphere with such extreme $t / R$ behaves as a homogeneous sphere [32]. On the contrary, the static friction coefficient at $t / R$ between the extreme $t / R$ ranges increases with the normal load (e.g., $\mu$ as a function of $P^{*}$ at $t / R$ denoted by the vertical dashed line II). In this $t / R$ range, the coated sphere exhibits a feature unique to the coated spherical contact, i.e., the stress level in the coating is relieved by the plastically deformed substrate [23]. As the normal load increases, the substrate becomes more plastic and compliant and relieves the stress level in the coating. This results in a more elastic coating, and hence, a junction capable of supporting additional stress and a larger friction force.

Figure 8 shows the effect of $E_{\text {su }} / Y_{\text {sur }}$ where an increase in $E_{\mathrm{su}} / Y_{\mathrm{su}}$ increases $(t / R)_{\mathrm{m}}$ and $\mu_{\mathrm{m}}$. A higher $E_{\mathrm{su}} / Y_{\mathrm{su}}$ for a fixed $E_{\mathrm{su}}$ indicates a weaker substrate; hence, under a fixed normal load, the substrate plasticity level is higher. This provides a greater scope for the first mechanism indicated in Section 4.1 to be the dominant one, explaining the increase in $(t / R)_{\mathrm{m}}$ and $\mu_{\mathrm{m}}$ with the increase in $E_{\mathrm{su}} / Y_{\mathrm{su}}$. The difference

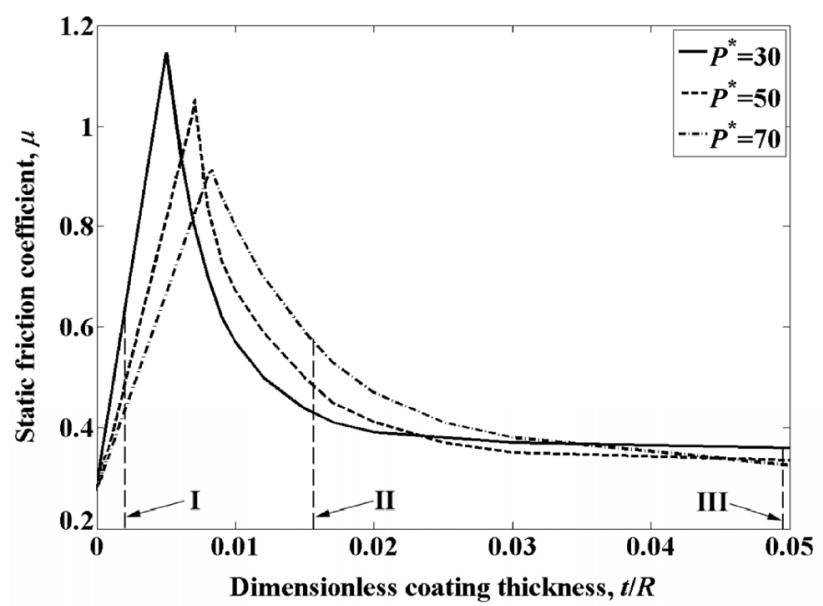

Fig. 7 Effect of normal loading on the static friction coefficient. 


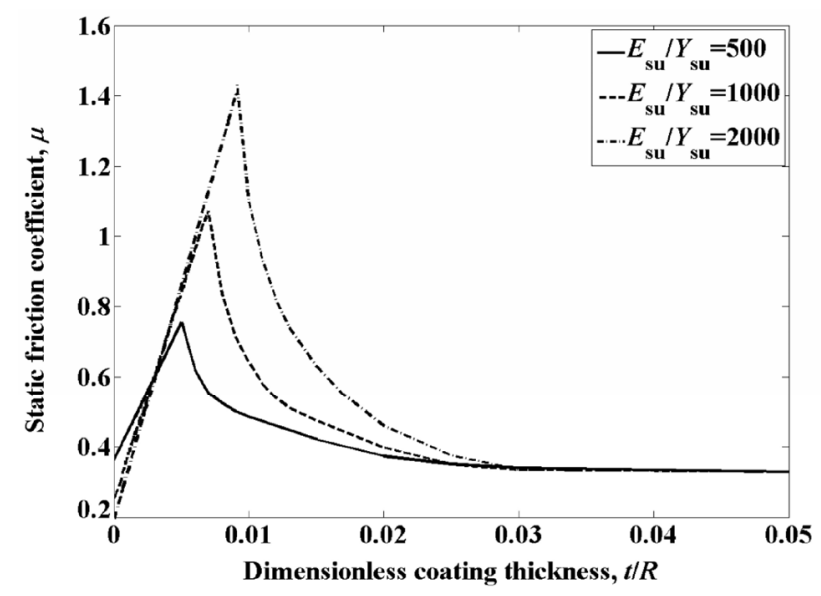

Fig. 8 Effect of $E_{\mathrm{su}} / Y_{\mathrm{su}}$ on the static friction coefficient.

between the three cases of $E_{\mathrm{su}} / Y_{\mathrm{su}}$ diminishes with the increase in $t / R$ as $\mu$ approaches the common $\mu_{\text {co }}$ because of the same coating material used in these cases.

Figure 9 shows the effect of $E_{\mathrm{co}} / E_{\mathrm{su}}$ where the static friction coefficient increases with $E_{\mathrm{co}} / E_{\mathrm{su}}$. A higher $E_{\mathrm{co}} / E_{\mathrm{su}}$ for a fixed $E_{\mathrm{su}}$ indicates a stiffer coating. This enables the junction to support additional stress, and hence, a larger friction force. In addition, $(t / R)_{\mathrm{m}}$ is independent of $E_{\mathrm{co}} / E_{\mathrm{su}}$.

From the results of the parametric analysis, it was observed that the effect of a decrease in $E_{\mathrm{co}} / Y_{\mathrm{co}}$ has the same trend as that shown in Fig. 9 for an increase in $E_{\mathrm{co}} / E_{\mathrm{su}}$. Similarly, $(t / R)_{\mathrm{m}}$ is independent of $E_{\mathrm{co}} / Y_{\mathrm{co}}$.

\subsection{A model for the static friction coefficient}

Extensive numerical simulations must be performed in order to obtain the expression of the static friction

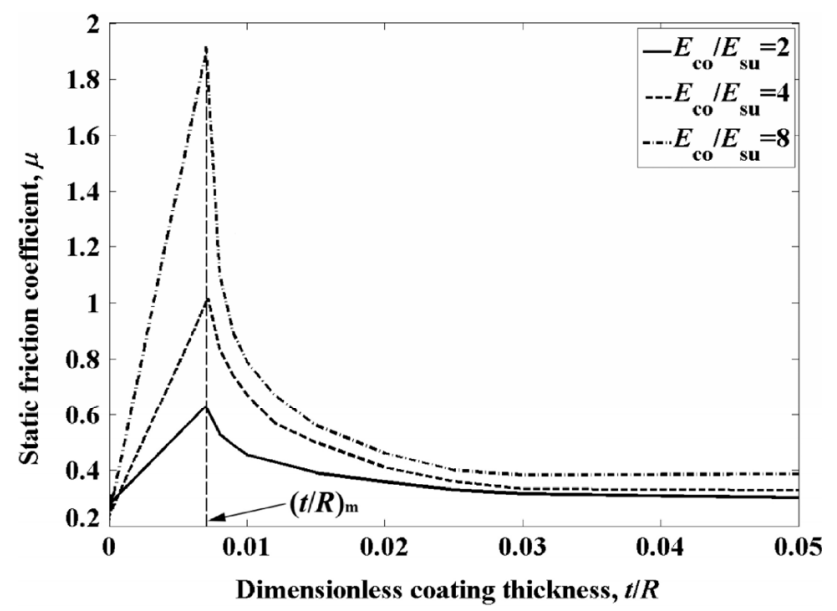

Fig. 9 Effect of $E_{\mathrm{co}} / E_{\mathrm{su}}$ on the static friction coefficient. coefficient in terms of the material and geometrical properties and normal load covering ranges indicated in Section 4.2. Table 1 presents the values of $E_{\mathrm{co}} / E_{\mathrm{su}}$ $E_{\mathrm{co}} / Y_{\mathrm{co},} E_{\mathrm{su}} / Y_{\mathrm{su}} P^{*}$, and $t / R$ used for the numerical simulations. Four material property combinations that result in $Y_{\mathrm{co}} / Y_{\mathrm{su}}<1$ (see section 4.2 and Ref. [16]) are eliminated. Thus, we have $(3 \times 3 \times 3-4) \times 5=115$ combinations of material properties and normal load, for each of which we obtained 12 values of $\mu$ corresponding to the 12 values of $t / R$. Consequently, the total number of data points used for obtaining the following empirical expressions (Eqs. (7-13)) is $115 \times 12=1380$.

It was observed that, for all the combinations of material properties and normal load, the behavior of $\mu$ with the increase in $t / R$ was similar to that shown in Fig. 3. Hence, the expression of $\mu$ should contain two parts for the two branches of $t / R$ divided by $(t / R)_{\mathrm{m}}$ and also ensure that $\mu=\mu_{\mathrm{su}}$ at $t / R=0$ and $\mu$ approaches $\mu_{\mathrm{co}}$ at large $t / R$. An admissible form for the expression of $\mu$ is as follows:

for $t / R \leq(t / R)_{\mathrm{m}}$

$$
\mu=\mu_{\mathrm{su}}+k\left(\frac{t}{R}\right)
$$

for $t / R>(t / R)_{\mathrm{m}}$

$$
\mu=\mu_{\mathrm{co}} \operatorname{coth}\left(a\left(\frac{t}{R}\right)^{b}\right)
$$

where $k$ is the slope of the linear function that fits the increasing $\mu$, and $a$ and $b$ are the fitting parameters inside the cotangent hyperbolic function that fits the decreasing $\mu$. Using Eqs. (7) and (8) to fit each of the 115 sets of numerical $\mu-t / R$ data (see Fig. 3 as an example), we observed that the $R^{2}$ goodness of fit is better than 0.9 for more than $80 \%$ of the sets (maximum error 15\%) and better than 0.8 for the rest (maximum error $30 \%$ ). Hence, such a form for the

Table 1 Values of the input dimensionless parameters.

\begin{tabular}{cc}
\hline$E_{\mathrm{co}} / E_{\mathrm{su}}$ & $2,4,8$ \\
\hline$E_{\mathrm{co}} / Y_{\mathrm{co}}$ & $500,1000,2000$ \\
$E_{\mathrm{su}} / Y_{\mathrm{su}}$ & $500,1000,2000$ \\
$P^{*}$ & $20,30,50,70,100$ \\
$t / R$ & $0.001,0.003,0.005, \ldots, 0.015,0.02,0.03,0.04,0.05$ \\
\hline
\end{tabular}


expression of $\mu$ based on Eqs. (7) and (8) was adopted. Accordingly, $(t / R)_{\mathrm{m}}$ and $\mu_{\mathrm{m}}$ corresponding to the transition of increasing $\mu$ to decreasing $\mu$ can be conveniently obtained as the intersection of the two branches of fitting curves for each set of numerical $\mu-t / R$ data. Their expressions are given by Eqs. (9) (maximum error 10\%) and (10) (maximum error 15\%), both of which have $R^{2}$ goodness of fit better than 0.97 .

$$
\begin{gathered}
(t / R)_{\mathrm{m}}=7.59 e-5\left(P^{*}\right)^{0.493}\left(E_{\mathrm{su}} / Y_{\mathrm{su}}\right)^{0.364} \\
\mu_{\mathrm{m}}=40.2\left(P^{*}\right)^{-0.209}\left(E_{\mathrm{co}} / E_{\mathrm{su}}\right)^{0.669}\left(E_{\mathrm{co}} / Y_{\mathrm{co}}\right)^{-0.875}\left(E_{\mathrm{su}} / Y_{\mathrm{su}}\right)^{0.332}
\end{gathered}
$$

As expected, $(t / R)_{\mathrm{m}}$ is a function of only $P^{*}$ and $E_{\mathrm{su}} / Y_{\mathrm{su}}$ whereas $\mu_{\mathrm{m}}$ depends on all the dimensionless parameters (see Section 4.2). As $\left((t / R)_{\mathrm{m}}, \mu_{\mathrm{m}}\right)$ must satisfy both Eqs. (7) and (8), the slope $k$ in Eq. (7) can be obtained from points $\left(0, \mu_{\mathrm{su}}\right)$ and $\left((t / R)_{\mathrm{m}}, \mu_{\mathrm{m}}\right)$ as follows:

$$
k=\frac{\mu_{\mathrm{m}}-\mu_{\mathrm{su}}}{(t / R)_{\mathrm{m}}}
$$

and a relation between the fitting parameters $a$ and $b$ can also be obtained by substituting $\left((t / R)_{\mathrm{m}}, \mu_{\mathrm{m}}\right)$ into Eq. (8) as follows:

$$
a=\frac{\operatorname{coth}^{-1}\left(\mu_{\mathrm{m}} / \mu_{\mathrm{co}}\right)}{(t / R)_{\mathrm{m}}^{b}}
$$

The parameter $b$ can be handily obtained from the fitting curves for each set of numerical $\mu-t / R$ data, whose expression is given by Eq. (13) with $R^{2}$ goodness of fit equal to 0.974 and a maximum error of $15 \%$.

$b=23.9\left(P^{*}\right)^{-0.195}\left(E_{\mathrm{co}} / E_{\mathrm{su}}\right)^{0.318}\left(E_{\mathrm{co}} / Y_{\mathrm{co}}\right)^{-0.693}\left(E_{\mathrm{su}} / Y_{\mathrm{su}}\right)^{0.298}$

Therefore, using Eqs. (7-13) along with Eqs. (2), (3), (5), and (6), which calculate $\mu_{\mathrm{co}}$ and $\mu_{\mathrm{su}}$ a model can be obtained for the static friction coefficient in a full stick elastic-plastic coated spherical contact. Notably, as the elastic-plastic model produces permanent distortion of the surface, the current model would only be valid for sliding of a new surface. Relaxing this limitation is a task for future work.
It was revealed in Ref. [40] that, with regard to critical loads at yield inception vs. dimensionless coating thickness, the behavior of soft coatings resembles a mirror image of the behavior of hard coatings. We thus speculate that soft coatings may provide a similar mirror image behavior with regard to the static friction coefficient vs. $t / R$ shown in Fig. 3. We used the finite element model to obtain $\mu$ for a single case of soft coating with $E_{\mathrm{co}} / E_{\mathrm{su}}=0.25, E_{\mathrm{co}} / Y_{\mathrm{co}}=$ $E_{\mathrm{su}} / Y_{\mathrm{su}}=1,000$ (resulting in $Y_{\mathrm{co}} / Y_{\mathrm{su}}=\left(E_{\mathrm{su}} / Y_{\mathrm{su}}\right)\left(E_{\mathrm{co}} / E_{\mathrm{su}}\right) /$ $\left.\left(E_{\mathrm{co}} / Y_{\mathrm{co}}\right)=0.25\right)$ and $P^{*}=50$ with $t / R$ from 0.001 to 0.05 . The results showed that as $t / R$ increased, $\mu$ decreased from $\mu_{\mathrm{su}}$ to a minimum and thereafter increased and approached $\mu_{\mathrm{co}}$. This single case resembles a mirror image of the behavior of $\mu$ with hard coatings. An intensive study over a large range of mechanical properties for soft coatings and the minimization of friction is very interesting but out of the scope of the present paper. This shall be covered in future work.

\section{Conclusion}

A finite element model was developed to investigate an elastic-plastic coated spherical contact under combined normal and tangential loading in the full stick contact condition. In this model, sliding inception was assumed to occur once the tangential stiffness became equal to or less than $10 \%$ of the initial tangential stiffness.

It was observed that, for fixed coating and substrate material properties and normal loading, the static friction coefficient $\mu$ is a function of the dimensionless coating thickness $t / R$. An intensive study was performed on hard coatings when $Y_{\mathrm{co}} / Y_{\mathrm{su}}>1$. As a typical behavior of such a case, $\mu$ first increases linearly with $t / R$ till reaching a maximum $\mu_{\mathrm{m}}$ at $t / R=(t / R)_{\mathrm{m}}$, and thereafter decreases as a cotangent hyperbolic function when $t / R$ increases above $(t / R)_{\mathrm{m}}$. When $t / R$ is 0 or very large, $\mu$ approaches the static friction coefficient $\mu_{\mathrm{su}}$ or $\mu_{\mathrm{co}}$ of a homogeneous sphere made of the substrate or coating material, respectively.

A parametric study was performed over a wide range of the dimensionless material properties $E_{\mathrm{co}} / E_{\mathrm{sw}}$ $E_{\mathrm{co}} / Y_{\mathrm{co}}$ and $E_{\mathrm{su}} / Y_{\mathrm{su}}$ and the dimensionless normal load $P^{*}$ to reveal the effects of these parameters on the static friction coefficient $\mu$ as a function of $t / R$. An 
increase in $E_{\mathrm{co}} / E_{\mathrm{su}}$ or a decrease in $E_{\mathrm{co}} / Y_{\mathrm{co}}$ increases $\mu$ for the entire range of $t / R$. The effect of $E_{\mathrm{su}} / Y_{\mathrm{su}}$ and $P^{*}$ is more complex as an increase in $P^{*}$ or $E_{\mathrm{su}} / Y_{\mathrm{su}}$ can either increase or decrease $\mu$ depending on $t / R$.

A model for the static friction coefficient $\mu$ in the case of hard coatings was proposed based on the typical behavior of $\mu$ as a function of $t / R$. The potential for mirror image behavior in the case of soft coatings that can minimize $\mu$ was also demonstrated.

\section{Acknowledgment}

This paper is part of IEA AMT IA technical activities.

Open Access: The articles published in this journal are distributed under the terms of the Creative Commons Attribution 4.0 International License (http:// creativecommons.org/licenses/by/4.0/), which permits unrestricted use, distribution, and reproduction in any medium, provided you give appropriate credit to the original author(s) and the source, provide a link to the Creative Commons license, and indicate if changes were made.

\section{References}

[1] Eriksson M, Bergman F, Jacobson S. On the nature of tribological contact in automotive brakes. Wear 252(1-2): 26-36 (2002)

[2] Ost W, De Baets P, Degrieck J. The tribological behaviour of paper friction plates for wet clutch application investigated on SAE\#II and pin-on-disk test rigs. Wear 249(5-6): 361-371 (2001)

[3] Srivastava N, Haque I. Transient dynamics of metal V-belt CVT: Effects of band pack slip and friction characteristic. Mech Mach Theory 43(4): 459-479 (2008)

[4] Salib J, Kligerman Y, Etsion I. A model for potential adhesive wear particle at sliding inception of a spherical contact. Tribol Lett 30(3): 225-233 (2008)

[5] Holmberg K, Andersson P, Erdemir A. Global energy consumption due to friction in passenger cars. Tribol Int 47: 221-234 (2012)

[6] Hintermann H E. Adhesion, friction and wear of thin hard coatings. Wear 100(1-3): 381-397 (1984)

[7] Holmberg K, Ronkainen H, Matthews A. Tribology of thin coatings. Ceram Int 26(7): 787-795 (2000)

[8] Hogmark S, Jacobson S, Larsson M. Design and evaluation of tribological coatings. Wear 246(1-2): 20-33 (2000)
[9] Holmberg K, Ronkainen H, Laukkanen A, Wallin K. Friction and wear of coated surfaces - scales, modelling and simulation of tribomechanisms. Surf Coat Technol 202(4-7): 1034-1049 (2007)

[10] Greenwood J, Williamson J B P. Contact of nominally flat surfaces. Proc Roy Soc A: Math, Phys Eng Sci 295(1442): 300-319 (1966)

[11] Keer L M, Kim S H, Eberhardt A W, Vithoontien V. Compliance of coated elastic bodies in contact. Int $J$ Solids Struct 27(6): 681-698 (1991)

[12] Garjonis J, Kačianauskas R, Stupak E, Vadlūga V. Investigation of contact behaviour of elastic layered spheres by FEM. Mechanika 3(77): 5-12 (2009)

[13] Goltsberg R, Etsion I. A universal model for the loaddisplacement relation in an elastic coated spherical contact. Wear 322-323: 126-132 (2015)

[14] Goltsberg R, Etsion I. Contact area and maximum equivalent stress in elastic spherical contact with thin hard coating. Tribol Int 93: 289-296 (2016)

[15] Goltsberg R, Etsion I, Davidi G. The onset of plastic yielding in a coated sphere compressed by a rigid flat. Wear 271(11-12): 2968-2977 (2011)

[16] Chen Z, Goltsberg R, Etsion I. Yield modes in a coated spherical contact. Tribol Int 120: 309-316 (2018)

[17] Goltsberg R, Etsion I. A model for the weakening effect of very thin hard coatings. Wear 308(1-2): 10-16 (2013)

[18] Huang X, Kasem H, Shang H F, Shao T M, Etsion I. Experimental study of a potential weakening effect in spheres with thin hard coatings. Wear 296(1-2): 590-597 (2012)

[19] Eid H, Joshi N, McGruer N E, Adams G G. A model of contact with adhesion of a layered elastic-plastic microsphere with a rigid flat surface. $J$ Tribol 133(3): 031406 (2011)

[20] Chen L, Guo Z J, Joshi N, Eid H, Adams G G, McGruer N E. An improved SPM-based contact tester for the study of microcontacts. J Micromech Microeng 22(4): 045017 (2012)

[21] Nguyen H V, He J Y, Helland T, Kristiansen H, Aasmundtveit $\mathrm{K}$ E. Electrical characterization of individual metal-coated polymer spheres used in isotropic conductive adhesives. $J$ Appl Polym Sci 133(31): 43764 (2016)

[22] Bazilchuk M, Pettersen S R, Kristiansen H, Zhang Z L, He $\mathrm{J}$ Y. Electromechanical characterization of individual micronsized metal coated polymer particles. J Appl Phys 119(24): 245102 (2016)

[23] Chen Z, Goltsberg R, Etsion I. Plasticity evolution in a coated sphere compressed by a rigid flat. Tribol Int $\mathbf{9 8}$ : 116-124 (2016)

[24] Chen Z, Goltsberg R, Etsion I. A universal model for a frictionless elastic-plastic coated spherical normal contact with moderate to large coating thicknesses. Tribol Int 114: 485-493 (2017) 
[25] Ronen S, Goltsberg R, Etsion I. A comparison of stick and slip contact conditions for a coated sphere compressed by a rigid flat. Friction 5(3): 326-338 (2017)

[26] Kogut L, Etsion I. A semi-analytical solution for the sliding inception of a spherical contact. J Tribol 125(3): 499-506 (2003)

[27] Eriten M, Polycarpou A A, Bergman L A. Physics-based modeling for partial slip behavior of spherical contacts. Int J Solids Struct 47(18-19): 2554-2567 (2010)

[28] García J M, Martini A. Measured and predicted static friction for real rough surfaces in point contact. $J$ Tribol 134(3): 031501 (2012)

[29] Wu A Z, Shi X, Polycarpou A A. An elastic-plastic spherical contact model under combined normal and tangential loading. J Appl Mech 79(5): 051001 (2012)

[30] Wu A Z, Shi X. Numerical investigation of adhesive wear and static friction based on the ductile fracture of junction. $J$ Appl Mech 80(4): 041032 (2013)

[31] Shi X. On slip inception and static friction for smooth dry contact. J Appl Mech 81(12): 121005 (2014)

[32] Brizmer V, Kligerman Y, Etsion I. Elastic-plastic spherical contact under combined normal and tangential loading in full stick. Tribol Lett 25(1): 61-70 (2007)

[33] Zolotarevskiy V, Kligerman Y, Etsion I. The evolution of static friction for elastic-plastic spherical contact in pre-sliding.

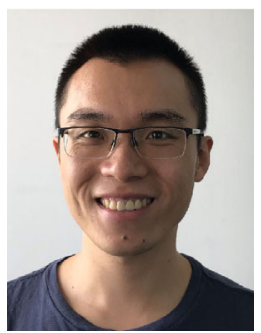

Zhou CHEN. He received his bachelor degree in mechanical engineering in 2014 from Harbin Institute of Technology, China. He is currently

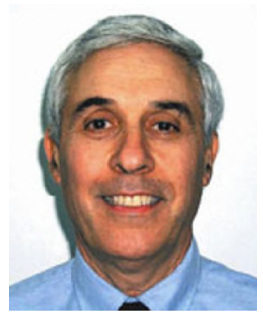

Izhak ETSION. He received his Ph.D degree in 1974 from TechnionIsrael Institute of Technology. Since then he was a faculty at the Department of Mechanical Engineering where he is currently a professor emeritus. His research interests are in hydrodynamic lubrication, surface texturing, contact mechanics, nano-tribology and bio-tribology. He
J Tribol 133(3): 034502 (2011)

[34] Bhagwat P, Sista B, Vemaganti K. A computational study of the effects of strain hardening in micro-asperity friction models. Tribol Lett 65(4): 154 (2017)

[35] Zhao B, Zhang S, Keer L M. Spherical elastic-plastic contact model for power-law hardening materials under combined normal and tangential loads. J Tribol 139(2): 021401 (2017)

[36] Etsion I. Revisiting the Cattaneo-Mindlin Concept of interfacial slip in tangentially loaded compliant bodies. J Tribol 132(2): 020801 (2010)

[37] Ovcharenko A, Halperin G, Etsion I. In situ and real-time optical investigation of junction growth in spherical elasticplastic contact. Wear 264(11-12): 1043-1050 (2008)

[38] ANSYS $^{\circledR}$ Academic Teaching Mechanical and CFD, Release 18.1, Help System. ANSYS Documentation/Mechanical APDL/Command References/XXIII. V Commands/VROTAT. ANSYS, Inc.

[39] ANSYS $^{\circledR}$ Academic Teaching Mechanical and CFD, Release 18.1, Help System. ANSYS Documentation/Mechanical APDL/Contact Technology Guide/3. Surface-to-Surface Contact (Pair-Based)/3.6 Defining the Target Surface/3.6.1 Pilot nodes. ANSYS, Inc.

[40] Goltsberg R, Levy S, Kligerman Y, Etsion I. Strengthening Effect of soft thin coatings. $J$ Tribol 140(6): 064501 (2018)

a Ph.D student in the Department of Mechanical Engineering, Technion, Israel. His research interests include contact mechanics and coatings.

has published over 200 papers on various aspects of tribology and holds 15 patents. He is the founder of Surface Technologies Ltd. that developed the laser surface texturing (LST) technology for friction and wear reduction. His honors include fellow of the ASME (1999), fellow of the STLE (2001), the STLE 2005 International Award and the ASME 2016 Mayo D. Hersey Award. 